\title{
Zur Theorie der Sättigung bei der magnetischen Kernresonanz
}

\author{
Von G. Vojta \\ Aus dem Theoretisch-Physikalischen Institut der Universität Leipzig \\ (Z. Naturforschg. 12 a, 282-294 [1957] ; eingegangen am 22. Dezember 1956)
}

\begin{abstract}
Zur theoretischen Untersuchung der Sättigungserscheinungen bei der magnetischen Kernresonanz wird eine allgemeine Bewegungsgleichung für die quantenstatistische Dichtematrix eines KernspinSystems hergeleitet und für den stationären Zustand gelöst. Dabei wird die Spin-Gitter-Wechselwirkung durch ein besonderes Relaxationsglied summarisch erfaßt. Kompliziertere Fälle, wie solche mit Quadrupol-Relaxation und Linienstrukturen durch Spin-Spin-Wechselwirkung, bleiben vorerst unberücksichtigt. Durch Berechnung des Erwartungswertes der HF-Magnetisierung ergeben sich Real- und Imaginärteil der HF-Suszeptibilität des Kernspin-Systems und damit Dispersion und Absorption. Der Fall gleicher Relaxationszeiten $T_{1}, T_{2}$ wird für zirkular wie für linear polarisiertes HF-Magnetfeld direkt behandelt, der Fall $T_{1} \neq T_{2}$ für das zirkular polarisierte HF-Magnetfeld mittels unitärer Transformation auf ein rotierendes Koordinatensystem, dessen $z$-Achse dauernd in Richtung des Gesamt-Magnetfeldes zeigt. Die Lösungen sind unter den gemachten Voraussetzungen für beliebige Feldstärken des konstanten und des HF-Magnetfeldes gültig, und zwar auch für elektronenparamagnetische Resonanzen. - Aus der Bewegungsgleichung für die Dichtematrix lassen sich modifizierte Buochsche Gleichungen ableiten, bei denen die Relaxation in bezug auf das in jedem Zeitpunkt gerade vorhandene Gesamt-Magnetfeld vor sich geht.
\end{abstract}

\section{Einleitung}

Bei der Untersuchung der kernmagnetischen Resonanzerscheinungen ist es - wie allgemein in der Hochfrequenz-Spektroskopie - wichtig, das Verhalten der Kernsignale unter Sättigungsbedingungen zu kennen, d. h. bei großer Amplitude des eingestrahlten HF-Magnetfeldes. Die ursprünglichen klassischen Bewegungsgleichungen für den Magnetisierungsvektor eines Spin-Systems, die sog. Blochschen Gleichungen ${ }^{1}$, geben in einfachen Fällen (vor allem bei Abwesenheit von Linienfeinstrukturen) die experimentellen Ergebnisse oft in guter Näherung wieder, und zwar auch für stärkere HF-Magnetfelder. Trotzdem ist natürlich eine quantenmechanische Theorie der Kernresonanz erwünscht. Von WangsNESS und BLOCH ${ }^{2}$ wurde unter recht einschränkenden Voraussetzungen eine quantenmechanische Rechtfertigung der BLochschen Gleichungen gegeben. Allerdings sind diese Gleichungen auch für die einfachsten Systeme (einatomige Gase) nicht streng richtig; u. a. liefern sie nicht die Debyesche Formel für die Absorption im Grenzfalle verschwindenden konstanten Magnetfeldes.

Die bisherigen theoretischen Ansätze zur Erklärung der Sättigungserscheinungen (welche auch den

1 F. Bloch, Phys. Rev. 70, 460 [1946].

2 R. K. Wangsness u. F. Bloch, Phys. Rev. 89, 728 [1953].

3 R. Karplus u. J. Schwinger, Phys. Rev. 73, 1020 [1948].

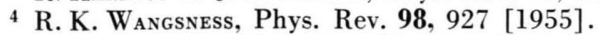

5 M. A. Garstens, Phys. Rev. 93, 1228 [1954].

6 M. A. Garstens u. J. I. Kaplan, Phys. Rev. 99, 459 [1955].

7 A. G. Redfield, Phys. Rev. 98, 1787 [1955]. eben angedeuteten Punkt mit zu berücksichtigen hätten) weisen u. a. eine oder mehrere der folgenden Beschränkungen auf:

a) Die Rechnung wird nur für Gase durchgeführt (hierbei ist die transversale Relaxationszeit $T_{2}$ gleich der longitudinalen Relaxationszeit $\left.T_{1}\right)^{3,4}$, teilweise ohne Benutzung der Quantentheorie ${ }^{5,6}$.

b) Es wird nur der Fall zirkular polarisierten HF-Magnetfeldes durchgerechnet, nicht der allgemeinere mit linear polarisiertem HF-Magnetfeld.

c) Die Gültigkeit der Rechnungen beschränkt sich auf sehr starke HF-Magnetfelder (und Festkörper) ${ }^{7}$.

d) Nur das Absorptionssignal $v=-\chi^{\prime \prime} H_{1}$ wird direkt berechnet, hingegen nicht das Dispersionssignal $u=\chi^{\prime} H_{1}\left(\chi=\chi^{\prime}-i \chi^{\prime \prime}\right.$ : komplexe HF-Suszeptibilität des Kernspin-Systems, $H_{1}$ : halbe Amplitude des eingestrahlten linear polarisierten HF-Magnetfeldes) ${ }^{8,9.10}$.

Das Absorptionssignal und damit $\chi^{\prime \prime}$ kann [vgl. d) ] durch Berechnung der vom Kernspin-System absorbierten HF-Leistung auch für starke $H_{1}$-Felder in einer vom quantentheoretischen Standpunkt befriedigenden Weise erhalten werden. Es wäre deshalb am einfachsten, $\chi^{\prime}$ aus $\chi^{\prime \prime}$ mit Hilfe der allgemeinen Kronig-Kramersschen Integralrelationen ${ }^{11,12,13} \mathrm{zu}$

8 N. Bloembergen, E. M. Purcell u. R. V. Pound, Phys. Rev. 73, 679 [1948].

9 N. Bloembergen, Dissertation, Leiden 1948.

10 A. M. Portis, Phys. Rev. 91, 1071 [1953].

11 R. DE L. Kronig, J. Opt. Soc. Amer. und Rev. Sci. Instrum. 12, 547 [1926].

12 M. H. A. Kramers, Atti Congr. Fisici, Como, 1927, 545.

13 E. Hiedemann u. R. D. Spence, Z. Phys. 133, 109 [1952]. 
ermitteln ${ }^{8,9}$, von denen die eine für Kernresonanz lautet:

$$
\chi^{\prime}(\omega)=\frac{1}{\pi} \int_{-\infty}^{\infty} \frac{\chi^{\prime \prime}(x)}{x-\omega} \mathrm{d} x .
$$

Indessen versagen die Kronig-Kramers-Relationen, sobald $\chi$ eine Funktion von $H_{1}$ wird, d. h., sobald die Beziehung zwischen HF-Magnetfeld und Kernmagnetisierung nicht mehr linear ist; das ist aber für nicht mehr verschwindend kleine $H_{1}$-Feldstärken der Fall ${ }^{10}$.

Naheliegend ist nun, eine Verallgemeinerung der Kronig-Kramers-Relationen für den nichtlinearen Fall zu versuchen. Das liefe auf eine nichtlineare Theorie der Relaxation hinaus, eine solche ist aber in allgemeiner Weise wahrscheinlich nicht durchführbar. (Eine Anwendung der linearen Relaxationstheorie auf den Kernresonanzfall wurde schon von Kuво und Tомгта ${ }^{14}$ vorgenommen.) - Wir haben nach einem Vorschlag von Lösche versucht, unter einschränkenden Voraussetzungen, teils auch unter Verwendung anderer Kerne als des obigen Cauchyschen Kernes, nutzbare Integralbeziehungen zwischen $\chi^{\prime}$ und $\chi^{\prime \prime}$ aufzustellen, aber vergebens. $\mathrm{Da}$ es offenbar kaum möglich ist, in befriedigender Weise (vgl. aber ${ }^{10}$ ) allgemein $\chi^{\prime}$ aus $\chi^{\prime \prime}$ abzuleiten - wir stimmen darin mit Brachman und MaCDONALD ${ }^{15}$ überein -, muß ein direkter Weg zur quantenmechanischen Berechnung von $\chi^{\prime}$ eingeschlagen werden.

Das Ziel der vorliegenden Arbeit ist eine so weit wie möglich allgemeine und einfache quantenmechanische Behandlung der Sättigungserscheinung und darüber hinaus der Dynamik eines KernspinSystems für beliebige Feldstärken des konstanten und des hochfrequenten Magnetfeldes im stationären („eingeschwungenen“) Zustand. Die Spin-GitterWechselwirkung wird durch ein besonderes Relaxationsglied in der Bewegungsgleichung für die Dichtematrix des Systems (s. u.) summarisch dargestellt. Verwickeltere Fälle, wie solche mit Linienstrukturen infolge Spin - Spin-Wechselwirkung oder Relaxation durch Quadrupol-Kopplung (soweit sie eine wesentliche Rolle spielt und nicht durch das Relaxationsglied mit erfaßt wird) und ebenso Erscheinungen wie der Overhauser-Effekt bleiben vorerst außer

14 R. Kuвo u. K. Tomita, J. Phys. Soc., Japan 9, 888 [1954].

15 Briefwechsel mit dem Verfasser.

16 K. Halbach, Helv. Phys. Acta 27, 259 [1954] ; 29, 37 [1956].

17 G. Vojta, Diplomarbeit, Leipzig 1955 (unveröffentlicht).
Betracht; eine weiterführende Untersuchung ist im Gange. Außerdem werden Komplikationen, wie sie z. B. durch Verwendung eines Autodyn-Detektors entstehen, nicht erörtert; dasselbe gilt von den Modulationseffekten, welche bereits in einer Reihe von Arbeiten erschöpfend behandelt worden sind $7,10,16,17$.

\section{Aufstellung einer allgemeinen Bewegungs- gleichung für die Dichtematrix eines Kernspinsystems}

Das geeignete Werkzeug zur Behandlung eines gegebenen thermodynamischen Systems (quantenstatistisch: einer gemischten Gesamtheit), wie es in der zu untersuchenden Probe von Kernen vorliegt, ist der Dichtematrix-Formalismus (s. z. B. ${ }^{18,19}$ ).

In einem zeitlich konstanten, homogenen Magnetfeld $H_{0}$ (in $z$-Richtung) befinde sich ein einzelner Kern mit der Spinquantenzahl $I$ und dem HamiltonOperator $\mathcal{H}^{(0)}=\mathcal{H}\left(H_{0}\right)=-\vec{\mu} \cdot \mathfrak{H}_{0}=-\gamma \hbar \mathfrak{\Im} \cdot \mathfrak{S}_{0}$ mit der Wahrscheinlichkeit $a_{m}(t) a_{m}^{*}(t)$ zur Zeit $t$ im Zustande mit der magnetischen Quantenzahl $m$, also mit der Energie

$$
E\left(H_{0}\right)_{m}=-\gamma \hbar H_{0} m .
$$

Dabei ist

$\vec{\mu}=\gamma \hbar$ s: $\quad$ Operator des magnetischen Dipolmoments des Kernes,

ə $=\left\{I_{x}, I_{y}, I_{z}\right\}:$ vektorieller Spinoperator (,Spinvektor"),

$\gamma: \quad$ gyromagnetisches Verhältnis des Kernes; es sei stets $\gamma>0$ angenommen.

Die Dichtematrix $\varrho(t)$ dieses Kernspins ist dann in der „Energiedarstellung“ (in $\operatorname{der} I_{z}$ und $\mathcal{H}^{(0)}$ diagonal sind) gegeben durch

$$
\begin{array}{cc} 
& \varrho_{m m^{\prime}}(t)=a_{m}(t) a_{m l^{\prime}}^{*}(t) \\
\text { mit } & \operatorname{Sp} \varrho(t)=\sum_{m} \varrho_{m m}(t)=1 .
\end{array}
$$

Es gilt die Bewcgungsgleichung (im SchrödingerBild)

$$
-\frac{\hbar}{i} \frac{\partial \varrho}{\partial t}=[\mathcal{H}, \varrho]=\mathcal{H} \supseteq-\varrho \mathcal{H},
$$

hierbei ist $\mathcal{H}$ allgemein der Hamilton-Operator des vorgelegten quantenmechanischen Systems, in unse-

18 J.v. Neumann, Nachr. d. Gesellschaft d. Wiss., Göttingen 1927, S. 273.

19 R. C. Tolman, Principles of Statistical Mechanics, Oxford University Press, London 1950, Kap. IX. 
rem Falle also $\mathcal{H}=\mathcal{H}^{(0)}$. Diese Bewegungsgleichung ist mit $\mathcal{H}=\mathcal{H}^{(0)}$ gleichbedeutend mit der unitären Transformation

$$
\varrho(t)=U^{+} \varrho(0) U
$$

mit $U=\exp \left[(i / \hbar) \mathcal{H}^{(0)} t\right] \quad$ (,Zeitverschiebungsoperator") und $U^{+}=U^{-1} ; U^{+}$ist der zu $U$ adjungierte Operator. Wegen der Larmor-Präzession des Kernspins entspricht diese Beziehung auch einem Übergang zu einem mit dem Kernspin mitrotierenden Koordinatensystem, in dem die Dichtematrix $\tilde{\varrho}$ gegeben ist durch

$$
\tilde{\varrho}(t)=U \varrho(t) U^{+} .
$$

Denn ist allgemein $\Omega$ ein quantenmechanischer Operator im Ausgangskoordinatensystem, dann ist dieser Operator in einem um die $z$-Achse um den Winkel $\varphi$ in positiver Richtung gedrehten Koordinatensystem zu schreiben

$$
\tilde{\Omega}=\exp \left[(i / \hbar) \varphi \mathcal{L}_{z}\right] \cdot \Omega \cdot \exp \left[-(i / \hbar) \varphi \mathcal{L}_{z}\right],
$$

wobei $\mathcal{L}_{z}$ der Operator der $z$-Komponente des Drehimpulses des vorliegenden Systems ist ${ }^{20,21}$.

Ist $\bar{A}$ der Erwartungswert irgend einer zu einem (nicht explizit zeitabhängigen) Spinoperator $\mathcal{A}$ gehörigen Spinfunktion $A$, so wird

$$
\overline{A(t)}=\mathrm{Sp}[\varrho(t) \cdot \mathcal{A}]=\operatorname{Sp}[\mathcal{A} \cdot \varrho(t)] ;
$$

hierbei ist $\mathcal{A}$ in der gewählten Darstellung zu nehmen, in der $I_{z}$ diagonal ist. Wir haben im folgenden insbesondere die $x$-Komponente und die $y$-Komponente der Kernmagnetisierung zu berechnen; z. B. ist

$$
M_{x}(t)=N \cdot \operatorname{Sp}\left[\mu_{x} \cdot \varrho(t)\right]=N \gamma \hbar \cdot \operatorname{Sp}\left[I_{x} \cdot \varrho(t)\right]
$$

( $N$ : Zahl der Kerne pro $\mathrm{cm}^{3}$ der Kernprobe). Denn wegen

$$
\begin{aligned}
& M_{x}=u \cos \omega t-v \sin \omega t, \\
& M_{y}=-u \sin \omega t-v \cos \omega t
\end{aligned}
$$

$(\omega$ : Kreisfrequenz des eingestrahlten HF-Magnetfeldes) ergeben sich z. B. aus $\overline{M_{x}}$ dann $\bar{u}$ und $\bar{v}$ und damit $\chi^{\prime}$ und $\chi^{\prime \prime}$.

Befindet sich im $H_{0}$-Feld ein System von freien, d. h. nicht miteinander in Wechselwirkung stehenden, gleichen Kernspins in einem Zustand, der for-

20 E. C. Kemble, Fundamental Principles of Quantum Mechanics, McGraw-Hill Book Comp., New York u. London 1937, S. 247 u. 307.

21 I. I. Rabi, N. F. Ramsey u. J. Schwinger, Rev. Mod. Phys. 26, 167 [1954]. mal einem thermodynamischen Gleichgewichtszustand bei einer Temperatur $T_{\mathrm{S}}$ („Spintemperatur“) entspricht, dann gilt für die - statistische! Gleichgewichts-Dichtematrix $\varrho_{0}$ eines der Kerne [vgl. (2.1) und (2.2)]:

$\varrho_{0}=\exp \left[-\mathcal{H}^{(0)} / k T_{\mathrm{S}}\right] / \mathrm{Sp} \exp \left[-\mathcal{H}^{(0)} / k T_{\mathrm{S}}\right]$,

wobei also jetzt die Nichtdiagonalelemente entsprechend dem diesbezüglichen Grundpostulat der Quantenstatistik ${ }^{19}$ gleich Null gesetzt sind (vgl. unten). Im allgemeinen (außer für sehr tiefe Temperaturen $T_{\mathrm{S}}$ oder/und sehr hohe Feldstärken) ist für Kernspins $E\left(H_{0}\right) \ll k T_{S}$; man kann deshalb in sehr guter Näherung setzen

$$
\exp \left[-\mathcal{H}^{(0)} / k T_{\mathrm{S}}\right]=\mathbf{1}-\mathcal{H}^{(0)} / k T_{\mathrm{S}}
$$

(1 ist die Einheitsmatrix).

Wir betrachten jetzt eine vorliegende Kernprobe, d. h. ein System von gleichen Kernspins im zugehörigen Gitter in einem $H_{0}$-Magnetfeld. Mit dem Begriff "Gitter" sei (auch für Gase und Flüssigkeiten) die molekulare Umgebung der Kerne mit sämtlichen (insbesondere den thermischen) Freiheitsgraden bezeichnet, welche nicht durch $\mathcal{H}^{(0)}$ erfaßt werden; die magnetischen Momente benachbarter Kerne werden nur soweit in Rechnung gesetzt, als sie einen Teil des Wärmereservoirs bilden. Dann genügt es, einen der Kerne herauszugreifen und ihn und seine Umgebung (Gitter) zu betrachten. In einer Darstellung, in der neben dem Hamilton-Operator $\mathcal{H}^{(0)}$ des Kernspins (Zustände durchnumeriert mit $m$ ) auch der Hamilton-Operator $\mathcal{H}_{\mathrm{G}}$ des Gitters (Zustände durchnumeriert mit einer Quantenzahl $g{ }^{*}$ ) diagonal ist, wird die Dichtematrix $\varrho_{(\mathrm{Sp}+\mathrm{G})}(t)$ unseres Systems (Kern und Umgebung) analog (2.1) definiert durch

$$
\varrho_{m g, m^{\prime} g^{\prime}}(t)=a_{m g}(t) a_{m^{\prime} g^{\prime}}^{*}(t) .
$$

Wir zeigen, daß bei unserer vereinfachten Behand. lung das Gitter nicht mit in die Rechnung eingeht. Vorerst sei der Hamilton-Operator der Spin - GitterWechselwirkung $\mathcal{H}_{\mathrm{W}}$ klein angenommen:

$$
\mathcal{H}_{\mathrm{W}} \ll \mathcal{H}^{(0)}, \mathcal{H}_{\mathrm{G}} \text {. }
$$

Mit $\varrho_{\mathrm{G}}: \varrho_{g g^{\prime}}(t)=\alpha_{g}(t) \alpha_{g^{\prime}}^{*}(t)$ als Dichtematrix des Gitters gilt dann

$$
\varrho_{m g, m^{\prime} g^{\prime}}=a_{m} a_{m^{\prime}}^{*} \cdot \alpha_{g} \alpha_{g^{\prime}}^{*}=\varrho_{m m^{\prime}} \varrho_{g g^{\prime}},
$$

* Das Gitter - bzw. das Gesamtsystem (Spins + Gitter) hat als abgeschlossenes System ein diskretes Energiespektrum. Die sicher vorliegende Entartung ist leicht zu berücksichtigen, spielt aber für unsere grundsätzlichen Überlegungen keine Rolle. 
d. h. $\varrho_{(\mathrm{Sp}+\mathrm{G})}$ ist als Kronecker-Produkt (direktes Produkt) von $\varrho$ und $\varrho_{\mathrm{G}}$ zu schreiben:

$$
\varrho_{(\mathrm{Sp}+\mathrm{G})}=\varrho \times \varrho_{\mathrm{G}} \cdot
$$

Mit $\varrho^{\prime}=\varrho \times \mathbf{1}$ und $\varrho_{\mathrm{G}}{ }^{\prime}=\mathbf{1} \times \varrho_{\mathrm{G}}$ gilt auch

$$
\varrho_{(\mathrm{Sp}+\mathrm{G})}=(\varrho \times \mathbf{1}) \cdot\left(\mathbf{1} \times \varrho_{\mathrm{G}}\right)=\varrho^{\prime} \cdot \varrho_{\mathrm{G}}{ }^{\prime}=\varrho_{\mathrm{G}}{ }^{\prime} \cdot \varrho^{\prime} .
$$

Im thermodynamischen Gleichgewicht (des Gesamtsystems) ist nach (2.10)

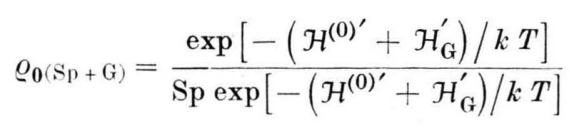

mit $\mathcal{H}^{(0)^{\prime}}=\mathcal{H}^{(0)} \times \mathbf{1}, \mathcal{H}_{\mathrm{G}}{ }^{\prime}=\mathbf{1} \times \mathcal{H}_{\mathrm{G}}$. Nun werde $\mathrm{zu}$ sätzlich ein (zirkular oder linear polarisiertes) HFMagnetfeld $\mathfrak{S}_{1}(t)$ eingestrahlt. Der gesamte $\mathrm{H}_{\text {AMIL- }}$ ToN-Operator des Systems (Kernspin + Gitter) ist mit $\mathcal{H}^{(1)}=-\gamma \hbar \mathfrak{J} \cdot \mathfrak{S}_{1}(t)$ jetzt zu schreiben

$$
\mathcal{H}=\mathcal{H}^{(0)}+\mathcal{H}^{(1)}+\mathcal{H}_{\mathrm{W}}+\mathcal{H}_{\mathrm{G}} \text {. }
$$

Wir nehmen an, daß das HF-Magnetfeld auf das Gitter nicht merklich einwirkt, ferner, daß das Gitter durch Zustandsänderungen der Spins an keiner Stelle wesentlich beeinflußt wird. Das Gitter wird also als „Wärmebad“ mit genügend großer Wärmekapazität (und genügend großer Wärmeleitfähigkeit) aufgefaßt. Wegen $E\left(H_{0}\right)+E\left(H_{1}\right) \ll k T$ ist das in sehr guter Näherung richtig. - Die allgemeine Bewegungsgleichung lautet jetzt

$$
-\frac{\hbar}{i} \frac{\partial}{\partial t} \varrho_{(\mathrm{Sp}+\mathrm{G})}=\left[\mathcal{H}, \varrho_{(\mathrm{Sp}+\mathrm{G})}\right],
$$

wobei für $\mathcal{H}$ zu schreiben ist

$$
\mathcal{H}^{\prime}=\mathcal{H}^{(0)^{\prime}}+\mathcal{H}^{(1)^{\prime}}+\mathcal{H}_{\mathrm{W}}{ }^{\prime}+\mathcal{H}_{\mathrm{G}}{ }^{\prime}
$$

mit $\mathcal{H}_{\mathrm{W}}{ }^{\prime}=\mathcal{H}_{\mathrm{W}} \times \mathbf{1}$, da das Gitter durch die SpinGitter-Wechselwirkung nicht merklich beeinflußt werden soll. Damit ist $\mathcal{H}_{\mathrm{W}}{ }^{\prime}$ ebenso wie $\mathcal{H}^{(0)^{\prime}}$ und $\mathcal{H}^{(1)^{\prime}}$ mit $\mathcal{H}_{\mathrm{G}}{ }^{\prime}$ und $\varrho_{\mathrm{G}^{\prime}}$ vertauschbar, entsprechend $\mathcal{H}_{\mathrm{G}}{ }^{\prime}$ mit $\varrho^{\prime}$, denn es ist z. B.

$$
\left[\mathcal{H}_{\mathrm{W}}{ }^{\prime}, \varrho_{\mathrm{G}}{ }^{\prime}\right]=\left(\mathcal{H}_{\mathrm{W}} \cdot \mathbf{1}\right) \times\left(\mathbf{1} \cdot \varrho_{\mathrm{G}}\right)-\left(\mathbf{1} \cdot \mathcal{H}_{\mathrm{W}}\right) \times\left(\varrho_{\mathrm{G}} \cdot \mathbf{1}\right)=0 .
$$

Da das Gitter als Wärmebad dauernd im thermischen Gleichgewicht beharren soll, fällt der Anteil $\varrho_{G}$ von $\varrho_{(\mathrm{Sp}+G)}$ heraus, was auch anschaulich klar ist, und wir haben nur die Dichtematrix $\varrho$ des Kernspins zu betrachten:

$$
\begin{aligned}
-\frac{\hbar}{i} \frac{\partial}{\partial t}\left(\varrho \times \varrho_{\mathrm{G}}\right) & =\left[\left(\mathcal{H}^{(0)}+\mathcal{H}^{(1)}+\mathcal{H}_{\mathrm{W}}\right) \times \mathbf{1}, \varrho \times \varrho_{\mathrm{G}}\right]+\left[\mathbf{1} \times \mathcal{H}_{\mathrm{G}}, \varrho \times \varrho_{\mathrm{G}}\right] \\
& =\left[\left(\mathcal{H}^{(0)}+\mathcal{H}^{(1)}+\mathcal{H}_{\mathrm{W}}, \varrho\right] \times \varrho_{\mathrm{G}}+\varrho \times\left[\mathcal{H}_{\mathrm{G}}, \varrho_{\mathrm{G}}\right] ;\right.
\end{aligned}
$$

daraus folgt

$$
-\frac{\hbar}{i} \frac{\partial \varrho}{\partial t}=\left[\mathcal{H}^{(0)}+\mathcal{H}^{(1)}+\mathcal{H}_{\mathrm{W}}, \varrho\right]
$$

Auch wenn jetzt nicht mehr $\mathcal{H}_{\mathrm{W}} \ll H^{(0)}, \mathcal{H}_{\mathrm{G}}$ gilt, bleibt wegen $\mathcal{H}_{\mathrm{W}}{ }^{\prime}=\mathcal{H}_{\mathrm{W}} \times \mathbf{1}$ der Gedankengang der gleiche. Die Gleichgewichts-Dichtematrix des Spinsystems wird zuerst

$$
\varrho_{0}=\text { const } \cdot \exp \left[-\left(\mathcal{H}^{(0)}+\mathcal{H}_{\mathrm{W}}\right) / k T\right] .
$$

Liegt ein Gas vor, kann $\mathcal{H}_{\mathrm{W}}$ einfach weggelassen werden (nur etwa während der Dauer eines Stoßes ist $\mathcal{H}_{\mathrm{W}} \neq 0$; die Wechselwirkungsenergie zweier Dipole ist indirekt proportional zur 3 . Potenz ihres Abstands). Im Falle einer Flüssigkeit ist der einfachste Ansatz für $\mathcal{H}_{\mathrm{W}}$ :

$$
\mathcal{H}_{\mathrm{W}}=-\gamma \hbar \mathfrak{J} \cdot \mathfrak{Y}_{\mathrm{loc}},
$$

wobei $\mathscr{Y}_{\text {loc }}$ das durch die thermische Bewegung des Gitters (z. B. der Flüssigkeitsmoleküle) hervorgerufene statistisch schwankende "lokale“ Magnetfeld am Ort des betrachteten Kernes ist. Das entspricht dem ersten Glied der üblichen „Multipol-Entwicklung “ der Wechselwirkungsenergie eines Kernes mit einem elektromagnetischen Feld, deren höhere Glieder vereinbarungsgemäß wegzulassen sind. Nun gilt aber für das Zeitmittel von $\mathcal{H}_{\mathrm{W}}$ :

$$
\overline{\mathcal{H}}_{\mathrm{W}}{ }^{t}=0 \text {. }
$$

Hier hat man über Zeiten. $t \gg T^{*}$ zu mitteln, wobei $T^{*}$ die Korrelationszeit der Flüssigkeit ist. Ist $T^{*}$ genügend klein (d. h. die Viskosität der Flüssigkeit genügend gering), so kann $t$ so klein gewählt werden, daß $\omega \ll 1 / t$. Betrachten wir nur jeweils über derartige Zeiten $t$ gemittelte Dichtematrizen, so kann $\mathcal{H}_{\mathrm{W}}$ in $\varrho_{0}$ wieder weggelassen werden. Für Festkörper läßt sich eine entsprechende Aussage höchstens in gewissen Fällen in grober Näherung machen (vgl. unten). 
Als Ausgangspunkt für die weitere Rechnung ist nun die Gleichung (2.12) (mit einem bestimmten Operator $\mathcal{H}_{\mathrm{W}}$ ) nicht brauchbar, da mit ihrer Hilfe ja kein zeitlich irreversibler Relaxationsvorgang beschrieben werden kann (man setze etwa $\mathcal{H}^{(1)} \doteq 0$ ). Bekannt ist aber, daß makroskopisch gesehen ein solcher Prozeß tatsächlich vor sich geht. Bezüglich des quantenstatistischen Formalismus zur Beschreibung des Spin - Gitter-Relaxationsmechanismus bzw. allgemein zur Beschreibung irreversibler Vorgänge verweisen wir auf eine folgende Arbeit. Hier gehen wir phänomenologisch vor und nehmen an, daß die Spin-Gitter-Wechselwirkung bei Abwesenheit des $H_{1}$-Feldes bei sprunghafter Änderung der Feldstärke des $H_{0}$-Feldes vom Wert $H_{0}{ }^{(0)}$ auf einen anderen Wert $H_{0}{ }^{(1)}$ eine zeitlich exponentiell verlaufende Annäherung der $z$-Komponente $M_{z}$ der Magnetisierung an den neuen Gleichgewichtswert $M_{z}{ }^{(1)}$ bewirkt gemäß der Gleichung

$$
M_{z}(t)=M_{z}^{(1)}+\left(M_{z}^{(0)}-M_{z}^{(1)}\right) e^{-t / T_{1}} .
$$

Das ist in sehr vielen Fällen bestimmt richtig. Es muß dann gelten

$$
\varrho(t)=\varrho_{0}{ }^{(1)}+\left(\varrho_{0}{ }^{(0)}-\varrho_{0}{ }^{(1)}\right) e^{-t / T_{1}} ;
$$

dies ergibt sich aber gerade als Lösung von

$$
\frac{\partial \varrho(t)}{\partial t}=-\frac{\varrho(t)-\varrho_{0}(1)}{T_{1}}
$$

mit $\varrho(0)=\varrho_{0}{ }^{(0)}$. Die letzten und alle folgenden Gleichungen gelten streng genommen für statistische Dichtematrizen $\bar{\varrho}$, bei denen über alle Kerne der Probe gemittelt ist; der Elementarvorgang bei der Relaxation wird natürlich nicht erfaßt (vgl. unten). Wir wollen aber trotzdem im allgemeinen weiter einfach $\varrho$ statt $\varrho$ schreiben. Vorerst sei $T_{1}=T_{2}=\tau$ gesetzt, auf den Fall $T_{1} \neq T_{2}$ und die transversale Relaxation gehen wir am Ende des Kapitels ein. Die Gleichheit beider Relaxationszeiten ist bekanntlich bei vielen Stoffen vorhanden, nämlich im allgemeinen bei Gasen und Flüssigkeiten geringer Viskosität.

Bei Anwesenheit eines (zirkular oder linear polarisierten) $H_{1}$-Feldes sei - im Gegensatz zu dem ursprünglichen BLochschen Ansatz - angenommen, daß die Relaxation in bezug auf das in jedem Zeitpunkt gerade vorhandene Gesamt-Magnetfeld vor sich geht; d. h. es gilt statt (2.14)

$$
\begin{array}{cc}
\frac{\partial \varrho(t)}{\partial t}=-\frac{\varrho(t)-\varrho_{0}(t)}{\tau} & \\
\text { mit } \quad \varrho_{0}(t)=\frac{\exp \left\{-\left[\mathcal{H}^{(0)}+\mathcal{H}^{(1)}(t)\right] / k T\right\}}{\operatorname{Sp} \exp \left\{-\left[\mathcal{H}^{(0)}+\mathcal{H}^{(1)}(t)\right] / k T\right\}}
\end{array}
$$

und

$$
\mathcal{H}^{(1)}(t)=-\gamma \hbar \mathfrak{\mathfrak { Y } _ { 1 }}(t),
$$

wobei für $\mathfrak{S}_{1}(t)$ entweder

$$
\begin{aligned}
& H_{x}=H_{1} \cos \omega t, \\
& H_{y}=-H_{1} \sin \omega t
\end{aligned}
$$

oder

$$
H_{x}=2 H_{1} \cos \omega t
$$

zu setzen ist. Allerdings ist diese "GleichgewichtsDichtematrix" $\varrho_{0}(t)$ keine strenge Lösung der Liouville-Gleichung (2.3), s. u. Damit entsteht endgültig (für $T_{1}=T_{2}$ ) folgende Bewegungsgleichung:

$$
\frac{\partial \varrho}{\partial t}=-\frac{i}{\hbar}\left[\mathcal{H}^{(0)}+\mathcal{H}^{(1)}(t), \varrho(t)\right]-\frac{\varrho(t)-\varrho_{0}(t)}{\tau} .
$$

Wir haben nun die Form der Gleichgewichts. Dichtematrix $\varrho_{0}(t)$ und des Relaxationsterms (2.15) näher zu begründen. $\varrho_{0}(t)$ in (2.16) ist nicht mehr diagonal, das steht im Widerspruch zu dem unter (2.10) erwähıten Grundpostulat der Quantenstatistik über die Gleichverteilung der Phasen der Wahrscheinlichkeitsamplituden in einer kanonischen Gesamtheit. Bei konsequentem Vorgehen hat man jedoch in einer Darstellung zu rechnen, in welcher der gesamte Hamilton-Operator $\mathcal{H}=\mathcal{H}^{(0)}+\mathcal{H}^{(1)}$ diagonal ist (wir können im Augenblick von der Spin Gitter-Wechselwirkung absehen und ein System von freien, nicht miteinander in Wechselwirkung stehenden Spins betrachten), also z. B. bei einem zirkular polarisierten $H_{1}$-Feld in einem mit diesem rotierenden Koordinatensystem. Dann ist auch die Matrix $\varrho_{0}=\exp [-\mathcal{H} / k T] / \mathrm{Sp} \exp [-\mathcal{H} / k T]$ wieder diagonal und das Postulat erfüllt. Die Frage ist nur, ob in einer solchen Darstellung dieses Postulat überhaupt sinnvoll ist.

Um die Sachlage näher zu untersuchen (und den Fall $T_{1} \neq T_{2}$ behandeln zu können, vgl. unten), diagonalisieren wir $\varrho_{0}(t)$ zu $\varrho_{0}$ mittels des Ansatzes

$$
\tilde{\varrho}_{0}=U \varrho_{0}(t) U^{+} \text {. }
$$

Der Einfachheit halber rechnen wir mit dem Spin $I=\frac{1}{2} . I_{x}, I_{y}, I_{z}$ werden dann durch die PAulischen Spinmatrizen gegeben, und mit $H_{x}=H_{1} \cos (\omega t)$, $H_{y}=-H_{1} \sin \omega t$ sowie $\delta_{0}=\gamma \hbar H_{0} / 2 k T$ und $\delta_{1}=\gamma \hbar H_{1} / 2 k T$ wird entsprechend (2.11)

$$
\varrho_{0}(t)=\frac{1}{2}\left(\begin{array}{cc}
1+\delta_{0} & \delta_{1} e^{i \omega t} \\
\delta_{1} e^{-i \omega t} & 1-\delta_{0}
\end{array}\right) .
$$

Für die unitäre Transformationsmatrix $U$ ergibt sich dann etwa: 


$$
U=\frac{1}{\sqrt{2} \delta_{01}}\left(\begin{array}{cc}
\sqrt{\delta_{01}+\delta_{0}} & \sqrt{\delta_{01}-\delta_{0}} \mathrm{e}^{i \omega t} \\
-\sqrt{\delta_{01}-\delta_{0} e^{-i \omega t}} & \sqrt{\delta_{01}+\delta_{0}}
\end{array}\right)
$$

mit $\delta_{01}=\sqrt{\delta_{0}{ }^{2}+\delta_{1}{ }^{2}}=\gamma \hbar \sqrt{H_{0}{ }^{2}+H_{1}{ }^{2}} / 2 k T$; weiter wird

$$
\tilde{\varrho}_{0}=\frac{\exp (-\tilde{\mathcal{H}} / k T)}{\operatorname{Sp} \exp (-\tilde{\mathcal{H}} / k T)}=\frac{1}{2}\left(\begin{array}{cc}
1+\delta_{01} & 0 \\
0 & 1-\delta_{01}
\end{array}\right) \text {, }
$$

wobei also

$$
\tilde{\mathcal{H}}=U\left(\mathcal{H}^{(0)}+\mathcal{H}^{(1)}\right) U^{+}=-\gamma \hbar \sqrt{H_{0}{ }^{2}+H_{1}{ }^{2} I_{z}}
$$

ist. Diese Transformation entspricht aber gerade dem Übergang auf ein Koordinatensystem $\tilde{\Sigma}$, dessen $\tilde{z}$-Achse dauernd in Richtung des Gesamtmagnetfeldes vom Betrag $\sqrt{{H_{0}}^{2}+H_{1}^{2}}$ (nicht des effektiven Magnetfeldes in einem um die ursprüngliche $z$-Achse rotierenden Koordinatensystem! - vgl. unten) zeigt.

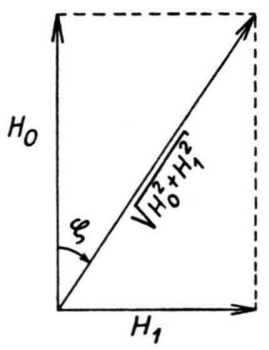

Abb. 1. Zur Transformation auf ein rotierendes Koordinatensystem.

Denn dieser Übergang wäre nach (2.6) mittels $U^{\prime}=U_{2} U_{1}$ auszuführen, wobei

$$
\begin{array}{ll}
U_{1}=e^{-i \omega t I_{z}} & (\text { Drehung "mit“ dem } \\
& \text { rotierenden Vektor } \left.\tilde{\mathfrak{S}}_{1}\right), \\
U_{2}=e^{i \zeta I_{y}} & (\text { Kippung um } \\
& \left.\zeta=\operatorname{arctg}\left[H_{1} / H_{0}\right]\right)
\end{array}
$$

ist. Für $I=\frac{1}{2}$ (für $I>\frac{1}{2}$ vgl. ${ }^{22}$ ) wird

$$
U_{1}=\left(\begin{array}{cc}
e^{-\frac{1}{2} i \omega t} & 0 \\
0 & e^{\frac{1}{2} i \omega t}
\end{array}\right), \quad U_{2}=\left(\begin{array}{rr}
\cos (\zeta / 2) & \sin (\zeta / 2) \\
-\sin (\zeta / 2) & \cos (\zeta / 2)
\end{array}\right)
$$

und

$$
U^{\prime}=U_{2} U_{1}=\left(\begin{array}{cc}
\cos \frac{\zeta}{2} e^{-\frac{1}{2} i \omega t} & \sin \frac{\zeta}{2} e^{\frac{1}{2} i \omega t} \\
-\sin \frac{\zeta}{2} e^{-\frac{1}{2} i \omega t} & \cos \frac{\zeta}{2} e^{\frac{1}{2} i \omega t}
\end{array}\right) .
$$

Durch Multiplikation der 1. Zeile mit $e^{\frac{1}{2} i \omega t}$ und der 2. Zeile mit $e^{-\frac{1}{2} i \omega t}$ geht $U^{\prime}$ in obiges $U$ über, wie

22 F. Bloch u. I. I. Rabi, Rev. Mod. Phys. 17, 237 [1945]. man leicht nachprüft. - Schließlich wird (2.17) zu

$$
\begin{gathered}
\frac{\partial \tilde{\varrho}}{\partial t}=-\frac{i}{\hbar}[\tilde{\mathcal{H}}, \tilde{\varrho}]+[V, \tilde{\varrho}]-\frac{\tilde{\varrho}-\tilde{\varrho}_{0}}{\tau} \\
\operatorname{mit} V=\frac{\partial U}{\partial t} U^{+} .
\end{gathered}
$$

Um vollkommene Analogie von (2.24) mit (2.17) herzustellen, hätte man noch einen „effektiven" Hamilton-Operator $\quad \tilde{\mathcal{H}}_{\mathrm{e}}=\tilde{\mathcal{H}}-(\hbar / i) V$ einzuführen (dieser wäre aber nicht mehr diagonal). REDField ${ }^{7}$ stellt in seiner Theorie, die u. a. nur für nicht zu kleine und nicht zu große $H_{1}$-Felder Gültigkeit hat, das Postulat auf, daß im stationären Zustand bei Einstrahlung des $H_{1}$-Feldes die Dichtematrix des Spinsystems durch $\exp \left[-\mathcal{H}_{\mathrm{er}} / k T\right]$ bestimmt wird, wobei $\mathcal{H}_{\mathrm{er}}=-\gamma \hbar \mathfrak{\Im} \cdot \mathfrak{\mathcal { g }}_{\mathrm{er}}(+$ Spin - Spin-Anteile $)$ der (nichtdiagonale) effektive Hamilton-Operator in einem um die ursprüngliche $z$-Achse rotierenden Koordinatensystem und $\mathfrak{\mathscr { S }}_{\mathrm{er}}$ dabei das effektive Magnetfeld $\left(H_{x}=H_{1}, H_{y}=0, H_{z}=H_{0}-\omega / \gamma\right)$ in diesem System ist. Dies kann nicht einwandfrei begründet werden und ist in verschiedener Hinsicht nicht streng gültig, u. a. ergibt sich damit die $\mathrm{Ab}$ sorption zu Null; ferner leidet dieser Versuch, Physik bzw. Statistik im rotierenden Koordinatensystem zu treiben, an der Inkonsequenz, daß die Relaxation nicht als durch $\mathfrak{H}_{\mathrm{er}}$ bestimmt angesehen werden kann, sondern - wie es der Wirklichkeit entspricht - durch $\mathscr{\mathfrak { S }}_{0}+\mathfrak{S}_{1}$ (oder $\mathfrak{S}_{0}$ für $H_{1} \ll H_{0}$ ).

Im Gegensatz zu REDFieLd transformieren wir aber nur aus zwei formalen Gründen auf unser rotierendes System $\tilde{\Sigma}$, deshalb ist bei uns auch $\tilde{\mathcal{H}}$ und nicht $\tilde{\mathcal{H}}_{\mathrm{e}}$ der maßgebende Намilton-Operator in $\widetilde{\Sigma}$, und die Relaxation geht richtig in bezug auf $H_{\tilde{z}}=\sqrt{{H_{0}}^{2}+{H_{1}}^{2}}$ vonstatten. Erstens wollen wir die nicht-diagonale Form von (2.16) begründen. Statt aber konsequent im System $\tilde{\Sigma}$ zu rechnen, können wir für $T_{1}=T_{2}$ im Laborsystem bleiben, da sich auf beiden Wegen dieselben Resultate ergeben. Zweitens ist der Fall $T_{1} \neq T_{2}$ nur im System $\tilde{\Sigma} \mathrm{zu}$ behandeln (s. unten). Da unsere Resultate mit experimentellen (und zum Teil auf andere Weise theoretisch erhaltenen) Ergebnissen übereinstimmen ${ }^{4,5,6}$, sehen wir unser Vorgehen als gerechtfertigt an.

Zur Begründung des Relaxationsterms (2.16) bemerken wir noch folgendes. Ein zeitlich exponentieller Verlauf der Relaxationsvorgänge gemäß (2.13) ist dann gegeben, wenn sich (für $H_{1} \ll H_{0}$ ) die Besetzungszahlen der einzelnen Energieniveaus $-\gamma \hbar H_{0} m$ so ändern, daß in jedem Zeitpunkt 
(einschl. $t=0$ ) eine Boltzmann-Verteilung mit einer Spintemperatur $T_{\mathrm{S}}$ besteht. Im Kernresonanzfall ist (für unser einfaches Kernspin-System und $H_{1} \ll H_{0}$ ) im stationären Zustand eine Boltzmann-Verteilung mit $T_{\mathrm{S}}>T$ vorhanden ${ }^{9}$. Die Besetzungszahlen sind aber gerade den Diagonalelementen der Dichtematrix proportional. Man zeigt durch Rechnen mit Übergangswahrscheinlichkeiten leicht, daß für den Vorgang $(2.12)$ z. B. die Unterschiede $\delta N_{i}$ der Besetzungszahlen der einzelnen Niveaus gegenüber den Gleichgewichtswerten der gleichen Differentialgleichung $\partial\left(\partial N_{i}\right) / \partial t=-\delta N_{i} / T_{1}$ gehorchen, wie die von uns unten verwendete Differenz-Dichtematrix $\Delta=\varrho-\varrho_{0}$.

Die Herleitung der Bewegungsgleichung (2.17) ist für elektronenparamagnetische Gase unter der Annahme starker und adiabatischer Stöße ${ }^{23}$ als Relaxationsmechanismus direkt möglich und bereits von Karplus und Schwinger ${ }^{3}$ durchgeführt worden; dabei ist $\tau$ die "mittlere" Zeit zwischen zwei Stößen. Diese Herleitung ist u. E. auch für den Fall der Kernresonanz in Gasen zu vertreten. Schwieriger liegen die Verhältnisse bei Flüssigkeiten; hier kann man vielleicht annehmen, daß nicht nach jedem einzelnen der „starken“ Stöße im Mittel BoltzmannVerteilung besteht, sondern jeweils erst nach einer Anzahl von Stößen. Bei Festkörpern ist mit entsprechenden Vorstellungen wohl kaum etwas anzufangen. Demgemäß beschränkt sich die Gültigkeit unserer Rechnungen vor allem auf (einfache) Gase (und zwar für Kernresonanz wie auch für elektronenparamagnetische Resonanz) sowie Flüssigkeiten (denen auch paramagnetische Ionen zugefügt sein können).

Wie erwähnt, ist die Gleichgewichts-Dichtematrix $\varrho_{0}(t)$ nach $(2.16)$ keine strenge Lösung der Liouville-Gleichung (2.3). Unter der Annahme der "Adiabasie" der Stoßprozesse stellt sie aber eine angenäherte Lösung dar:

$$
\partial \varrho / \partial t=0, \text { wenn } \omega \ll 1 / T^{*},
$$

dabei ist unter $T^{*}$ für Gase die Dauer eines Stoßprozesses (eigentlich die Dauer eines elementaren „Umklapp-Prozesses“ für einen Spin) zu verstehen, für Flüssigkeiten etwa die Korrelationszeit. Bezüglich der Stoßdauer bei Gasen ist man auf klassische (oder halbklassische) Abschätzungen angewiesen. Mit $R=$ Molekülradius und $v=$ Molekülgeschwindigkeit wird größenordnungsmäßig $T^{*}=R / v$. Für $R=10^{-8} \mathrm{~cm}$ und $v=500 \mathrm{~m} / \mathrm{sec}$ erhält man $1 / T^{*}=5 \cdot 10^{12} \mathrm{sec}^{-1}$, während z. B. für Kernreso. nanz mit Protonen bei einem Magnetfeld $H_{0}=10^{4} \mathrm{G}$ die Larmor-Frequenz $\omega_{0}=2,8 \cdot 10^{8} \mathrm{sec}^{-1}$ wird.

Aus der Dichtematrix-Gleichung (2.17) lassen sich unmittelbar modifizierte BLochsche Gleichungen für den Erwartungswert $\overline{\mathfrak{M}}$ des Kern-Magnetisierungsvektors $\mathfrak{M}$ mit den Komponenten $M_{x}, M_{y}, M_{z}$ ableiten. Dazu hat man nur beiderseits mit $N \gamma \hbar \mathfrak{J}$ zu multiplizieren und die Spur zu bilden. Es entsteht dann mit $\overline{M_{x}}=N \gamma \hbar \operatorname{Sp} I_{x} \varrho$ usw.

$$
\frac{\mathrm{d} \mathfrak{M}}{\mathrm{d} t}=\gamma \overline{\left[\mathfrak{M} \times \mathfrak{S}_{\mathfrak{C}}\right]}-\frac{\overline{\mathfrak{M}}-\overline{\mathfrak{M}_{0}}}{\tau},
$$

wobei sich das erste Glied rechts z. B. für die $x$ Komponente folgendermaßen ergibt:

$$
\begin{aligned}
-\frac{i}{\hbar} N \gamma \hbar \cdot \operatorname{Sp}\left[I_{x}(\mathcal{H}, \varrho)\right] & =-i N \gamma\left\{\operatorname{Sp} \varrho\left(I_{x} \mathcal{H}\right)-\operatorname{Sp} \varrho\left(\mathcal{H} I_{x}\right)\right\} \\
& =i N \gamma^{2} \hbar\left\{\overline{I_{x}(\mathfrak{J} \cdot \mathfrak{S})}-\overline{\left(\mathfrak{J} \cdot \mathfrak{S}_{2}\right) I_{x}}\right\}=\gamma(\mathfrak{M} \times \mathfrak{S})_{x} .
\end{aligned}
$$

Wir arbeiten aber weiterhin mit der DichtematrixGleichung, da sie allgemeiner ist als die BLochschen Gleichungen.

Abschließend sei noch der Fall ungleicher Relaxationszeiten $T_{1}, T_{2}$ betrachtet. Wir nehmen zuerst $H_{1} \ll H_{0}$ an, so daß die longitudinale Relaxation in bezug auf das $H_{0}$-Feld stattfindet, die transversale senkrecht dazu in der $x y$-Ebene. Wegen der Gestalt der Operatoren $I_{x}, I_{y}, I_{z}$ in der gewählten Darstellung wird aber die erstere nur durch die

23 J. H. van Vleck u. V. F. Weisskopf, Rev. Mod. Phys. 17, 227 [1945].
Diagonalelemente der Dichtematrix bestimmt, die letztere nur durch Nichtdiagonalelemente. Um jetzt also die richtige Bewegungsgleichung zu erhalten, hat man einfach im Relaxationsglied für die Diagonalelemente der Dichtematrix $T_{1}$ statt $\tau$ und für die Nichtdiagonalelemente $T_{2}$ statt $\tau$ zu schreiben, und folgerichtig ist dann

$$
\varrho_{0}=\exp \left[-\mathcal{H}^{(0)} / k T\right] / \mathrm{Sp} \exp \left[-\mathcal{H}^{(0)} / k T\right]
$$

zu setzen. Schwieriger liegen die Verhältnisse, wenn $H_{1} \ll H_{0}$ nicht mehr gilt und folglich die longitudinale Relaxation wie erwähnt in bezug auf das Ge- 
samt-Magnetfeld vor sich geht, die transversale Relaxation in einer Ebene senkrecht zu ihm. Hier hilft die Transformation auf ein rotierendes bzw. schwingendes Koordinatensystem, dessen $z$-Achse in jedem Zeitpunkt mit der Richtung des GesamtMagnetfeldes zusammenfällt, s. oben. Dann sind in diesem System die eben durchgeführten Überlegungen wieder gültig ${ }^{*}$.

\section{Lösung der Bewegungsgleichung für gleiche Relaxationszeiten $T_{1}, T_{2}$}

Wir suchen die stationäre Lösung der Gl. (2.17). Zur Rechenerleichterung führen wir die Matrix $\Delta$ ein mit

$$
\Delta(t)=\varrho(t)-\varrho_{0}(t) .
$$

Gl. (2.17) wird mit

$$
\begin{gathered}
{\left[\mathcal{H}^{(0)}+\mathcal{H}^{(1)}(t), \varrho_{0}(t)\right]=0 \mathrm{zu}} \\
\frac{\partial}{\partial t} \Delta(t)=-\frac{i}{\hbar}\left[\mathcal{H}^{(0)}+\mathcal{H}^{(1)}(t), \Delta(t)\right]-\frac{\Delta(t)}{\tau}-\frac{\partial \varrho_{0}(t)}{\partial t} .
\end{gathered}
$$

Der Einfachheit halber rechnen wir wieder mit $I=\frac{1}{2}$ und nehmen zuerst den Fall zirkular polarisierten $H_{1}$-Feldes: $H_{x}=H_{1} \cos \omega t, H_{y}=-H_{1} \sin \omega t$. Dann ist $\varrho_{0}(t)$ durch (2.19) gegeben. Aus (3.2) entsteht mit $\omega_{0}=\gamma H_{0}$ für die 4 Elemente von $\Delta$ :

$$
\left.\begin{array}{c}
\left(\frac{\partial}{\partial t}-i \omega_{0}+\frac{1}{\tau}\right) \Delta_{12}=\frac{1}{2} i \gamma H_{1} e^{i \omega t}\left(\Delta_{22}-\Delta_{11}\right)-\frac{1}{2} i \omega \delta_{1} e^{i \omega t}, \\
\left(\frac{\partial}{\partial t}+i \omega_{0}+\frac{1}{\tau}\right) \Delta_{21}=-\frac{1}{2} i \gamma H_{1} e^{-i \omega t}\left(\Delta_{22}-\Delta_{11}\right)+\frac{1}{2} i \omega \delta_{1} e^{-i \omega t}, \\
\left(\frac{\partial}{\partial t}+\frac{1}{\tau}\right) \Delta_{11}=\frac{1}{2} i \gamma H_{1}\left(\mathrm{e}^{i \omega t} \Delta_{21}-e^{-i \omega t} \Delta_{12}\right) .
\end{array}\right\}
$$

Es ist $\Delta_{22}=-\Delta_{11}$, weil $\operatorname{Sp} \Delta=0$ wegen $\operatorname{Sp} \varrho_{0}=1$ und $\operatorname{Sp} \varrho=1$, ferner ist $\Delta_{21}=\Delta_{12}{ }^{*}$.

Für die gesuchte stationäre Lösung machen wir, di. $\Delta_{11}, \Delta_{22}$ nur in $\overline{M_{z}}$ und $\Delta_{12}, \Delta_{21}$ nur in $\overline{M_{x}}, \overline{M_{y}}$ eingehen, den Ansatz

$$
\begin{gathered}
\Delta_{11}=R_{11}, \Delta_{12}=P_{12} e^{i \omega t}, \Delta_{21}=Q_{21} e^{-i \omega \mathrm{t}} \\
\left(Q_{21}=P_{12}{ }^{*}\right) .
\end{gathered}
$$

Nach kurzer Rechnung ergibt sich

$$
\begin{gathered}
R_{11}=-\frac{\frac{1}{2} \omega \delta_{1} \gamma H_{1} \tau^{2}}{1+\left(\omega_{0}-\omega\right)^{2} \tau^{2}+\gamma^{2} H_{1}{ }^{2} \tau^{2}}, \\
P_{12}=-\frac{1}{2} i \omega \delta_{1} \tau \frac{1+i\left(\omega_{0}-\omega\right) \tau}{1+\left(\omega_{0}-\omega\right)^{2} \tau^{2}+\gamma^{2} H_{1}{ }^{2} \tau^{2}} .
\end{gathered}
$$

Damit ist $\Delta(t)$ und $\varrho(t)$ ermittelt.

Wir berechnen $\overline{M_{x}}$ und $\overline{M_{z}}$. Nach (2.8) ist

$$
\overline{M_{x}}=N \gamma \hbar \operatorname{Sp}\left\{\left(\varrho_{0}+\Delta\right) I_{x}\right\} \text {, wobei }
$$

* Nach der obigen Diskussion unseres Ansatzes ist nicht von vornherein zu übersehen, inwieweit mit ihm Spinsysteme (Flüssigkeiten) mit $T_{1} \neq T_{2}$ behandelt werden können. Das Relaxationsglied ist gerechtfertigt, wenn man unter $T^{*}$ immer eine „Umklapp-Dauer“ für einen Spin verste-
$N \gamma \hbar \operatorname{Sp}\left(\varrho_{0} I_{x}\right)=\chi_{0} H_{1} \cos \omega t$,

$N \gamma \hbar \operatorname{Sp}\left(\Delta I_{x}\right)=\chi_{0} \omega \tau H_{1} \frac{\sin \omega t+\left(\omega_{0}-\omega\right) \tau \cos \omega t}{1+\left(\omega_{0}-\omega\right)^{2} \tau^{2}+\gamma^{2} H_{1}{ }^{2} \tau^{2}}$.

Mit (2.9) erhält man

$$
\begin{aligned}
& \chi^{\prime}=\chi_{0}+\chi_{0} \omega \tau \frac{\left(\omega_{0}-\omega\right) \tau}{1+\left(\omega_{0}-\omega\right)^{2} \tau^{2}+\gamma^{2} H_{1}^{2} \tau^{2}}, \\
& \chi^{\prime \prime}=\quad \chi_{0} \omega \tau \frac{1}{1+\left(\omega_{0}-\omega\right)^{2} \tau^{2}+\gamma^{2} H_{1}{ }^{2} \tau^{2}} .
\end{aligned}
$$

Weiter ist $\quad \overline{M_{z}}=N \gamma \hbar \mathrm{Sp}\left(\varrho_{0}+\Delta\right) I_{z}$,

mit $M_{z}^{(0)}=\chi_{0} H_{0}$ also

$$
\bar{M}_{z}=M_{z}{ }^{(0)}-\frac{\chi_{0} \omega \gamma H_{1}^{2} \tau^{2}}{1+\left(\omega_{0}-\omega\right)^{2} \tau^{2}+\gamma^{2} H_{1}^{2} \tau^{2}} .
$$

Es folgt der Fall linear polarisierten $H_{1}$-Feldes: $H_{x}=2 H_{1} \cos \omega t$. Man hat jetzt

$$
\varrho_{0}(t)=\frac{1}{2}\left(\begin{array}{cc}
1+\delta_{0} & 2 \delta_{1} \cos \omega t \\
2 \delta_{1} \cos \omega t & 1-\delta_{0}
\end{array}\right),
$$

hen darf; das bedeutet aber zweifellos eine Vergröberung des Sachverhalts. Vorbehaltlich einer genaueren Begründung sollen die betreffenden Rechnungen trotzdem durchgeführt werden. 
und damit entstehen folgende Gleichungen:

$$
\begin{aligned}
\left(\frac{\partial}{\partial t}-i \omega_{0}+\frac{1}{\tau}\right) \Delta_{12} & =i \gamma H_{1} \cos \omega t\left(\Delta_{22}-\Delta_{11}\right)+\omega \delta_{1} \sin \omega t, \\
\left(\frac{\partial}{\partial t}+i \omega_{0}+\frac{1}{\tau}\right) \Delta_{21} & =-i \gamma H_{1} \cos \omega t\left(\Delta_{22}-\Delta_{11}\right)+\omega \delta_{1} \sin \omega t, \\
\left(\frac{\partial}{\partial t}+\frac{1}{\tau}\right) \Delta_{11} & =i \gamma H_{1} \cos \omega t\left(\Delta_{21}-\Delta_{12}\right) ;
\end{aligned}
$$

hierbei ist wieder $\Delta_{21}=\Delta_{12}{ }^{*}$ und $\Delta_{22}=-\Delta_{11}$. Wir lösen sie mittels des Ansatzes

$$
\begin{array}{lll}
\Delta_{\mu \mu}=R_{\mu \mu} ; & \Delta_{\mu \nu}=P_{\mu \nu} e^{i \omega t}+Q_{\mu \nu} e^{-i \omega t}, & (\mu \neq v) \\
\mu, v=1,2 ; & Q_{\mu \nu}=P_{\nu \mu}^{*} . &
\end{array}
$$

Das bedeutet wiederum, daß wir $\overline{M_{z}}$ zeitlich konstant annehmen. Während diese Annahme bei zirku- lar polarisiertem $H_{1}$-Feld streng richtig ist, wird sie es bei linear polarisiertem $H_{1}$-Feld nicht sein. Die Gln. (3.10) sind mit dem Ansatz (3.11) auch nicht „in sich" lösbar, wir vernachlässigen aber einfach die beim Einsetzen von (3.11) in (3.10) auftretenden Terme mit $2 \omega$. Das Zeitmittel von $\overline{M_{z}}$ über eine Periode wird konstant sein, und mit diesem rechnen wir. Damit ergeben sich die Beziehungen

und daraus

$$
\begin{aligned}
& R_{11}=\frac{1}{2} i \gamma H_{1} \tau\left(P_{21}+Q_{21}-P_{12}-Q_{12}\right), \\
& P_{12}=\frac{-1}{(1 / \tau)-i\left(\omega_{0}-\omega\right)}\left(i \gamma H_{1} R_{11}+\frac{1}{2} i \omega \delta_{1}\right), \\
& P_{21}=\frac{1}{(1 / \tau)-i\left(\omega_{0}+\omega\right)}\left(i \gamma H_{1} R_{11}-\frac{1}{2} i \omega \delta_{1}\right), \\
& Q_{12}=P_{21}^{*}, \quad Q_{21}=P_{12}{ }^{*}
\end{aligned}
$$

$$
\begin{aligned}
& R_{11}=-\frac{\frac{1}{2} \omega \delta_{1} \gamma H_{1} \tau^{2}\left[\frac{1}{1+\left(\omega_{0}-\omega\right)^{2} \tau^{2}}-\frac{1}{1+\left(\omega_{0}+\omega\right)^{2} \tau^{2}}\right]}{1+\gamma^{2} H_{1}{ }^{2} \tau^{2}\left[\frac{1}{1+\left(\omega_{0}-\omega\right)^{2} \tau^{2}}+\frac{1}{1+\left(\omega_{0}+\omega\right)^{2} \tau^{2}}\right]}, \\
& P_{12}=-\frac{1}{2} i \omega \delta_{1} \tau \frac{1+i\left(\omega_{0}-\omega\right) \tau}{1+\left(\omega_{0}-\omega\right)^{2} \tau^{2}} \cdot \frac{1+\frac{2 \gamma^{2} H_{1}{ }^{2} \tau^{2}}{1+\left(\omega_{0}+\omega\right)^{2} \tau^{2}}}{1+\gamma^{2} H_{1}{ }^{2} \tau^{2}\left[\frac{1}{1+\left(\omega_{0}-\omega\right)^{2} \tau^{2}}+\frac{1}{1+\left(\omega_{0}+\omega\right)^{2} \tau^{2}}\right]}, \\
& P_{21}=-\frac{1}{2} i \omega \delta_{1} \tau \frac{1-i\left(\omega_{0}+\omega\right) \tau}{1+\left(\omega_{0}+\omega\right)^{2} \tau^{2}} \cdot \frac{2 \gamma^{2} H_{1}{ }^{2} \tau^{2}}{1+\left(\omega_{0}-\omega\right)^{2} \tau^{2}} \\
& R_{22}=-R_{11} ; \quad Q_{12}=P_{21}{ }^{*} ; \quad Q_{21}=P_{12}{ }^{*} H_{1}{ }^{2} \tau^{2}\left[\frac{1}{1+\left(\omega_{0}+\omega\right)^{2} \tau^{2}}+\frac{1}{1+\left(\omega_{0}-\omega\right)^{2} \tau^{2}}\right]
\end{aligned}
$$

Damit ist $\Delta(t)$ und $\varrho(t)$ für diesen allgemeineren Fall bekannt.

Als Anwendung berechnen wir wieder $\overline{M_{x}}$ und $\overline{M_{z}}$; ferner interessiert uns jetzt noch $\overline{M_{y}}$ für den Fall, daß im Experiment eine BLochsche Zweispulenanordnung verwendet wird. Es ist für $\overline{M_{x}}$ :

$$
N \gamma \hbar \cdot \operatorname{Sp}\left(\varrho_{0} I_{x}\right)=\chi_{0} \cdot 2 H_{1} \cos \omega t,
$$

$$
\begin{aligned}
& N \gamma \hbar \cdot \operatorname{Sp}\left(\Delta I_{x}\right)=\chi_{0} \omega \tau H_{1} \frac{1}{1+\gamma^{2} H_{1}{ }^{2} \tau^{2}\left[\frac{1}{1+\left(\omega_{0}-\omega\right)^{2} \tau^{2}}+\frac{1}{1+\left(\omega_{0}+\omega\right)^{2} \tau^{2}}\right]} . \\
& \quad\left\{\begin{array}{l}
\left.1+\frac{2 \gamma^{2} H_{1}{ }^{2} \tau^{2}}{1+\left(\omega_{0}+\omega\right)^{2} \tau^{2}}\left[\sin \omega t+\left(\omega_{0}-\omega\right) \tau \cos \omega t\right]+\frac{1+\frac{2 \gamma^{2} H_{1}{ }^{2} \tau^{2}}{1+\left(\omega_{0}-\omega\right)^{2} \tau^{2}}}{1+\left(\omega_{0}+\omega\right)^{2} \tau^{2}}\left[\sin \omega t-\left(\omega_{0}+\omega\right) \tau \cos \omega t\right]\right\} . \\
1+\left(\omega_{0}-\omega\right)^{2} \tau^{2}
\end{array} .\right.
\end{aligned}
$$


In dieser Schreibweise sieht man besonders deutlich, daß beim Vernachlässigen der Glieder mit dem Nenner $1+\left(\omega_{0}+\omega\right)^{2} \tau^{2}(3.14 \mathrm{~b})$ in $(3.6 \mathrm{~b})$ übergeht, ferner, daß für $\gamma^{2} H_{1}{ }^{2} \tau^{2} \rightarrow 0$ mittels $(2.9 \mathrm{a})$ z. B. der bekannte Ausdruck für $\chi^{\prime \prime}$

$$
\chi^{\prime \prime}=\frac{1}{2} \chi_{0} \omega \tau\left[\frac{1}{1+\left(\omega_{0}-\omega\right)^{2} \tau^{2}}+\frac{1}{1+\left(\omega_{0}+\omega\right)^{2} \tau^{2}}\right]
$$

entsteht. Weiter ergibt sich für $\overline{M_{y}}$ :

$$
\begin{aligned}
& N \gamma \hbar \cdot \operatorname{Sp}\left(\varrho_{0} I_{y}\right)=0, \\
& N \gamma \hbar \cdot \operatorname{Sp}\left(\Delta I_{y}\right)=\chi_{0} \omega \tau H_{1} \frac{1}{1+\gamma^{2} H_{1}{ }^{2} \tau^{2}\left[\frac{1}{1+\left(\omega_{0}-\omega\right)^{2} \tau^{2}}+\frac{1}{1+\left(\omega_{0}+\omega\right)^{2} \tau^{2}}\right]} . \\
& \left.\quad \cdot\left\{\frac{1+\frac{2 \gamma^{2} H_{1}^{2} \tau^{2}}{1+\left(\omega_{0}+\omega\right)^{2} \tau^{2}}}{1+\left(\omega_{0}-\omega\right)^{2} \tau^{2}}\left[\cos \omega t-\left(\omega_{0}-\omega\right) \tau \sin \omega t\right]-\frac{1+\frac{2 \gamma^{2} H_{1}^{2} \tau^{2}}{1+\left(\omega_{0}-\omega\right)^{2} \tau^{2}}}{1+\left(\omega_{0}+\omega\right)^{2} \tau^{2}}\left[\cos \omega t+\left(\omega_{0}+\omega\right) \tau \sin \omega t\right]\right\} .15 \mathrm{~b}\right)
\end{aligned}
$$

Die Glieder mit dem Nenner $1+\left(\omega_{0}+\omega\right)^{2} \tau^{2}$ werden gerade durch die „gegenläufige“ der beiden rotierenden Komponenten, in welche man das linear polarisierte $H_{1}$-Feld zerlegt denken kann, erzeugt. Vernachlässigt man sie, so erhält man mittels $(2.9 \mathrm{~b})$ wieder $(3.7 \mathrm{a}),(3.7 \mathrm{~b})$. Mit dem vollständigen Ausdruck (3.15) und $(2.9 \mathrm{~b})$ bekommt man aber andere Suszeptibilitäten $\chi^{\prime}, \chi^{\prime \prime}$ als mit (3.14b) [und (3.14a)] und $(2.9 \mathrm{a})$, was auch anschaulich verständlich ist. Aus $\overline{M_{x}}$ ergibt sich nämlich

$$
\begin{aligned}
& \chi^{\prime}=\chi_{0}+\chi_{0} \omega^{2} \tau^{2}\left[\left(\omega_{0}{ }^{2}-\omega^{2}\right) \tau^{2}-\left(1+2 \gamma^{2}{H_{1}}^{2} \tau^{2}\right)\right] \frac{1}{n}, \\
& \chi^{\prime \prime}=\quad \chi_{0} \omega \tau\left[1+\left(\omega_{0}^{2}+\omega^{2}\right) \tau^{2}+2 \gamma^{2}{H_{1}}^{2} \tau^{2}\right] \frac{1}{n},
\end{aligned}
$$

- wobei $\quad n=1+2\left(\omega_{0}^{2}+\omega^{2}\right) \tau^{2}+\left(\omega_{0}{ }^{2}-\omega^{2}\right)^{2} \tau^{4}+2 \gamma^{2} H_{1}^{2} \tau^{2}\left[1+\left(\omega_{0}{ }^{2}+\omega^{2}\right) \tau^{2}\right]-$

hingegen aus $\overline{M_{y}}$

$$
\begin{aligned}
& \chi^{\prime}=\chi_{0}+\chi_{0} \omega \omega_{0} \tau^{2}\left[\left(\omega_{0}^{2}-\omega^{2}\right) \tau^{2}+\left(1+2 \gamma^{2} H_{1}^{2} \tau^{2}\right)\right] \frac{1}{n} \\
& \chi^{\prime \prime}=\quad \chi_{0} \omega^{2} \omega_{0} \tau^{3} \frac{1}{n}
\end{aligned}
$$

Für $\overline{M_{z}}$ erhält man schließlich

$$
\overline{M_{z}}=M_{z}^{(0)}-4 \chi_{0} \omega_{0} \omega^{2} \gamma H_{1}{ }^{2} \tau^{4} \frac{1}{n} .
$$

Für zirkular polarisiertes $H_{1}$-Feld unterscheiden sich die von uns erhaltenen Suszeptibilitäten (3.7a), (3.7b) von den BLochschen Suszeptibilitäten - s. u. $(4.5 \mathrm{a}),(4.5 \mathrm{~b})$ - durch den Faktor $\omega$ (statt $\omega_{0}$ ) und durch das zusätzliche Glied $\chi_{0}$ in $\chi^{\prime}$. Dadurch geht für $\omega \rightarrow 0 \quad \chi^{\prime} \rightarrow \chi_{0}$ und $\chi^{\prime \prime} \rightarrow 0$, ferner geht $\bar{M}_{z} \rightarrow M_{z}{ }^{(0)}$. Für $\omega_{0}=\gamma H_{0} \rightarrow 0$ hat man

$$
\chi^{\prime} \rightarrow \chi_{0}\left(1+\gamma^{2} H_{1}^{2} \tau^{2}\right) /\left(1+\omega^{2} \tau^{2}+\gamma^{2} H_{1}^{2} \tau^{2}\right)
$$

und $\quad \chi^{\prime \prime} \rightarrow \chi_{0} \omega \tau /\left(1+\omega^{2} \tau^{2}+\gamma^{2} H_{1}{ }^{2} \tau^{2}\right)$

und erhält daraus für $\gamma^{2} H_{1}{ }^{2} \tau^{2} \rightarrow 0$ die Debyeschen Formeln für Dispersion und Absorption.

Für $\gamma^{2} H_{1}^{2} \tau^{2} \rightarrow 0$ sind auch die Kronig-Kramersschen Formeln erfüllt; wie man leicht nachrechnet, ergibt sich z. B. $\chi^{\prime}(\omega)$ gemäß (3.7 a) einschließlich des Gliedes $\chi_{0}$ mittels (1.1) aus $\chi^{\prime \prime}(\omega)$ nach $(3.7 \mathrm{~b})$.

Entsprechendes gilt für die Suszeptibilitäten für linear polarisiertes $H_{1}$-Feld. Man erhält z. B. für $\omega_{0} \rightarrow 0$ und $\gamma^{2} H_{1}{ }^{2} \tau^{2} \rightarrow 0$ aus (3.16a) und (3.16b) ebenfalls die Debyeschen Suszeptibilitäten

$$
\chi^{\prime}=\frac{\chi_{0}}{1+\omega^{2} \tau^{2}} \quad \text { und } \quad \chi^{\prime \prime}=\frac{\chi_{0} \omega \tau}{1+\omega^{2} \tau^{2}} ;
$$

hingegen wird in diesem Falle $\overline{M_{y}}$ und $\chi^{\prime}$ sowie $\chi^{\prime \prime}$ nach $(3.17 \mathrm{a}),(3.17 \mathrm{~b})$ zu Null, was auch zu fordern ist. Mittels der Kronig-Kramers-Relationen erhält man für $\gamma^{2} H_{1}^{2} \tau^{2} \rightarrow 0 \mathrm{z}$. B. aus $(3.14 \mathrm{c})$ wieder die richtige Dispersion

$$
\begin{aligned}
\chi^{\prime}(\omega)=\chi_{0}+\frac{1}{2} \chi_{0} \omega \tau \\
\cdot\left[\frac{\left(\omega_{0}-\omega\right) \tau}{1+\left(\omega_{0}-\omega\right)^{2} \tau^{2}}-\frac{\left(\omega_{0}+\omega\right) \tau}{1+\left(\omega_{0}+\omega\right)^{2} \tau^{2}}\right]
\end{aligned}
$$

zurück.

Das Maximum von $\chi^{\prime \prime}$, d. h. maximale Absorption, ergibt sich für zirkular polarisiertes $H_{1}$-Feld 
nach $(3.7 \mathrm{~b})$ bei fester Amplitude $H_{1}$ und fester Frequenz $\omega_{0}=\gamma H_{0}$ für die Resonanzfrequenz

$$
\omega_{\text {res }}=\omega_{0} \sqrt{1+\frac{1+\gamma^{2} H_{1}{ }^{2} \tau^{2}}{\omega_{0}^{2} \tau^{2}}} \approx \omega_{0}+\frac{1}{2} \frac{1+\gamma^{2} H_{1}{ }^{2} \tau^{2}}{\omega_{0} \tau^{2}}
$$

(für $\omega_{0}^{2} \tau^{2} \gg 1+\gamma^{2} H_{1}^{2} \tau^{2}$ ),

dagegen bei festgehaltener Frequenz $\omega$ und „Durchziehen " des $H_{0}$-Feldes (das entspricht oft dem Vorgehen im Experiment) für den Resonanzwert $H_{0 \mathrm{res}}=\omega / \gamma$. Das Minimum von $\overline{M_{z}}$ liegt nach (3.8) im ersten Falle bei der gleichen Resonanzfrequenz $\omega_{\text {res }}$, im zweiten Falle bei einem etwas kleineren Wert als $H_{0}$ res (die Berechnung führt auf eine Gleichung 4. Grades). Bei konstanter Frequenz $\omega$ ist das Maximum von $|v|$ gegeben durch

$$
|v|_{\max }=\chi_{0} \omega \tau \frac{H_{1}}{1+\gamma^{2} H_{1}^{2} \tau^{2}} .
$$

Die Funktion $|v|_{\max }\left(H_{1}\right)$ erreicht dann für eine optimale HF-Amplitude $H_{1 \text { opt }}$ mit $\gamma^{2} H_{1}{ }^{2}{ }_{\text {opt }} \tau^{2}=1$ ihren höchsten Wert $|v|_{\max \max }=\frac{1}{2} \chi_{0}(\omega / \gamma)$, s. Abb. 2 . Die Dispersionskurve $u=u\left(\omega_{0}\right)$ hat ihre Extrem. werte für $\omega=$ const, $H_{1}=$ const bei

$$
\omega_{0 \text { extr }}=\omega \pm \frac{1}{\tau} \sqrt{1+\gamma^{2} H_{1}^{2} \tau^{2}},
$$

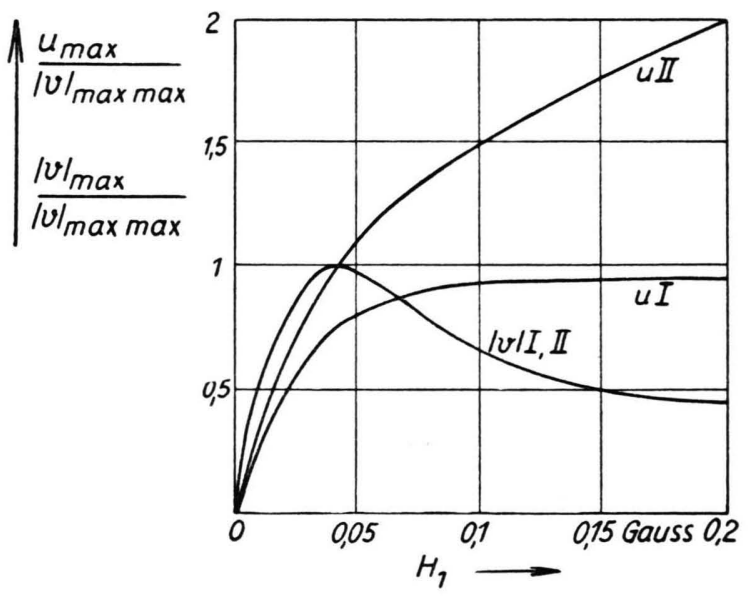

Abb. 2. Zum Sättigungsverhalten des ] ispersionssignals $u$ und des Absorptionssignals $v$ bei zirkular polarisiertem HF-Magnetfeld. Aufgetragen ist $u_{\max } /|v| \max \max$ und $|v|_{\max } /|v|_{\max \max }$ für zwei Fälle. Für das Beispiel wurden Protonen gewählt: $\gamma=2,675 \cdot 10^{4} \mathrm{G}^{-1} \mathrm{sec}^{-1}, \gamma_{0}=3,26 \cdot 10^{-10}$ bei $T=293^{\circ} \mathrm{K}$. Im Falle I ist $\omega=10^{8} \mathrm{sec}^{-1}, \tau=10^{-3} \mathrm{sec}$, im Falle II $\omega=10^{4} \mathrm{sec}^{-1}, \tau=10^{-3} \mathrm{sec}$. Im allgemeinen ist das Verhalten gemäß Fall I vorhanden, das Glied $\chi_{0} \cdot H_{1}$ im Ausdruck für $u$ spielt dann keine Rolle. Fall II ist etwa bei Untersuchungen wie denen von G. BÉnÉ, R. Extermann u. a. (Helv. Phys. Acta 28, 626 [1955]) verwirklicht (also bei schwachen $H_{0}$-Feldern). sie haben die Größe

$$
u_{\text {extr }}=\chi_{0} H_{1} \pm \frac{1}{2} \chi_{0} \omega \tau \frac{H_{1}}{\sqrt{1+\gamma^{2} H_{1}^{2} \tau^{2}}} .
$$

Für $H_{1} \rightarrow \infty$ nähert sich das zweite Glied dem Grenzwert $\frac{1}{2} \chi_{0}(\omega / \gamma)$, s. Abb. 2. Mit wachsender Amplitude $H_{1}$ des HF-Magnetfeldes wird also das Dispersionssignal wegen des Gliedes $\chi_{0} H_{1}$ ständig anwachsen. Betreffs einer ausführlichen Diskussion der Kurven $\chi^{\prime}(\omega)$ und $\chi^{\prime \prime}(\omega)$ vgl. Wangsness ${ }^{4}$.

In der Abb. 3 ist die Form der Kernsignale in Abhängigkeit von $\omega$ für verschiedene $H_{0}$-Werte angegeben, und zwar für ein zirkular polarisiertes
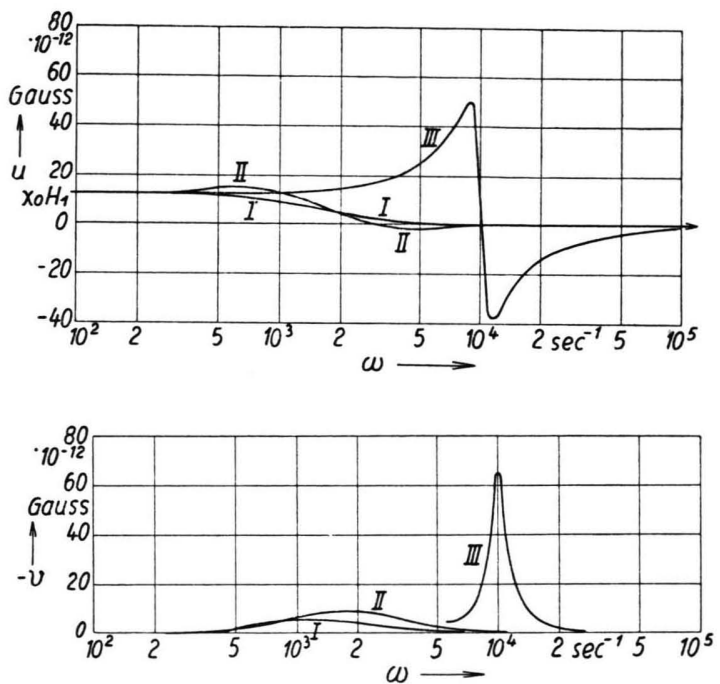

Abb. 3. Zum Übergang von reinem Relaxationsverhalten zum Resonanzverhalten bei zirkular polarisiertem HF-Magnetfeld. Beispiel: Protonen (vgl. Abb. 2) bei $H_{1}=0,040 \mathrm{G}$. I: $\omega_{0}=\gamma H_{0}=0$, II : $\omega_{0}=10^{3} \mathrm{sec}^{-1}$, III : $\omega_{0}=10^{4} \mathrm{sec}^{-1}$. Man beachte die Lage der Absorptionsmaxima.

HF-Magnetfeld bei konstanter Amplitude $H_{1}$. Zugrunde gelegt wurden die Gln. (3.7 a) und (3.7 b) (mit $\omega_{0}=\gamma H_{0}$ ), durch welche also das gesamte Verhalten des Spinsystems vom reinen Relaxationsfall $\left(H_{0}=0\right)$ bis zum Resonanzverhalten beherrscht wird. - Für linear polarisiertes $H_{1}$-Feld findet man Kurven für $\chi^{\prime}$ und $\chi^{\prime \prime}$ in Abhängigkeit von $\omega_{0}$ und für verschiedene $H_{1}$-Werte bei Garstens und Kaplan ${ }^{6}$, deren klassisch errechneten Ergebnisse mit unseren übereinstimmen.

\section{Der Fall $T_{1} \neq T_{2}$}

Unserem in Kap. 2 entwickelten Programm gemäß rechnen wir zuerst mit $H_{1} \ll H_{0}$ und müssen jetzt 
die Gl. (2.17) lösen, schreiben aber für $\varrho_{0}(t)$ $\varrho_{0}=\frac{\exp \left(-\mathcal{H}^{(0)} / k T\right)}{\operatorname{Sp} \exp \left(-\mathcal{H}^{(0)} / k T\right)}=\frac{1}{2}\left(\begin{array}{cc}1+\delta_{0} & 0 \\ 0 & 1-\delta_{0}\end{array}\right)$ und ersetzen für die Diagonalelemente der Dichtematrix $\tau$ durch $T_{1}$ und für die nichtdiagonalen Elemente $\tau$ durch $T_{2}$. Der Einfachheit halber rechnen wir mit zirkular polarisiertem $H_{1}$-Feld. Es ergeben sich folgende Gleichungen:

$$
\begin{aligned}
\left(\frac{\partial}{\partial t}-i \omega_{0}+\frac{1}{T_{2}}\right) \varrho_{12} & =\frac{1}{2} i \gamma H_{1} e^{i \omega t}\left(\varrho_{22}-\varrho_{11}\right), \\
\left(\frac{\partial}{\partial t}+i \omega_{0}+\frac{1}{T_{2}}\right) \varrho_{21} & =-\frac{1}{2} i \gamma H_{1} e^{-i \omega t}\left(\varrho_{22}-\varrho_{11}\right), \\
\left(\frac{\partial}{\partial t}+\frac{1}{T_{1}}\right) \varrho_{11} & =\frac{1}{2} i \gamma H_{1}\left(e^{i \omega t} \varrho_{21}-e^{-i \omega t} \varrho_{12}\right)+\frac{1+\delta_{0}}{2 T_{1}}, \\
\left(\frac{\partial}{\partial t}+\frac{1}{T_{1}}\right) \varrho_{22} & =-\frac{1}{2} i \gamma H_{1}\left(e^{i \omega t} \varrho_{21}-e^{-i \omega t} \varrho_{12}\right)+\frac{1-\delta_{0}}{2 T_{1}} .
\end{aligned}
$$

Ihre Lösung erfolgt wieder mittels eines Ansatzes:

$$
\varrho_{11}=r_{11}, \quad \varrho_{22}=r_{22}, \quad \varrho_{12}=p_{12} e^{i \omega t}, \quad \varrho_{21}=q_{21} e^{-i \omega t} .
$$

Man erhält schließlich

$$
\overline{M_{x}}=N \gamma \hbar \cdot \operatorname{Sp}\left(\varrho I_{x}\right)=\chi_{0} \omega_{0} T_{2} H_{1} \frac{\sin \omega t+\left(\omega_{0}-\omega\right) T_{2} \cos \omega t}{1+\left(\omega_{0}-\omega\right)^{2} T_{2}{ }^{2}+\gamma^{2} H_{1}{ }^{2} T_{1} T_{2}},
$$

also

$$
\begin{aligned}
& \chi^{\prime}=\chi_{0} \omega_{0} T_{2} \frac{\left(\omega_{0}-\omega\right) T_{2}}{1+\left(\omega_{0}-\omega\right)^{2} T_{2}^{2}+\gamma^{2} H_{1}^{2} T_{1} T_{2}}, \\
& \chi^{\prime \prime}=\chi_{0} \omega_{0} T_{2} \frac{1}{1+\left(\omega_{0}-\omega\right)^{2} T_{2}^{2}+\gamma^{2} H_{1}^{2} T_{1} T_{2}} .
\end{aligned}
$$

Das sind erwartungsgemäß gerade die BLochschen Suszeptibilitäten. - Man kann eine entsprechende Rechnung auch für ein linear polarisiertes $H_{1}$-Feld durchführen; wir vermerken hier nur, daß z. B. in $\overline{M_{x}}$ gegenüber der Formel (3.17) das Glied $\chi_{0} \cdot 2 H_{1} \cos \omega t$ wegfällt und der "Sättigungsparameter" $s=\gamma^{2} H_{1}{ }^{2} \tau^{2}$ durch $\gamma^{2} H_{1}{ }^{2} T_{1} T_{2}$ sowie "vorn" $\omega$ durch $\omega_{0} \mathrm{zu}$ ersetzen ist - es treten also die gleichen Unterschiede gegenüber śrüher auf, wie beim zirkular polarisierten $H_{1}$-Feld.
Wir lassen nunmehr die Beschränkung $H_{1} \ll H_{0}$ fallen und schreiben dazu die Gl. (3.2) für $\Delta$ in das rotierende Koordinatensystem $\tilde{\Sigma}$ um. Aus (2.24) folgt mit $\tilde{\Delta}=\tilde{\varrho}-\tilde{\varrho}_{0}$

$$
\frac{\partial \tilde{\Delta}}{\partial t}=-\frac{i}{\hbar}[\tilde{\mathcal{H}}, \tilde{\Delta}]+[V, \tilde{\Delta}]+\left[V, \tilde{\varrho}_{0}\right]-\frac{\tilde{\Delta}}{\tau}
$$

und daraus mittels $V=\frac{\partial U}{\partial t} U^{+}$und (2.20) und mit $\omega_{z}=\gamma \sqrt{H_{0}^{2}+H_{1}^{2}}$ sowie $\tau=T_{1}$ oder $T_{2}$

$$
\left(\frac{\partial}{\partial t}-i \omega_{z}+\frac{1}{T_{2}}\right) \tilde{\Delta}_{12}=\frac{i \omega}{2 \delta_{01}}\left\{2\left(\delta_{01}-\delta_{0}\right) \tilde{\Delta}_{12}+\delta_{1} e^{i \omega t}\left(\tilde{\Delta}_{22}-\tilde{\Delta}_{11}\right)\right\}-\frac{1}{2} i \omega \delta_{1} e^{i \omega t},
$$

die dazu konjugiert komplexe Gleichung für $\tilde{\Delta}_{21}=\tilde{\Delta}_{12}{ }^{*}$,

$$
\left(\frac{\partial}{\partial t}+\frac{1}{T_{1}}\right) \tilde{\Delta}_{11}=i \omega \frac{\delta_{1}}{2 \delta_{01}}\left\{e^{i \omega t} \tilde{\Delta}_{21}-e^{-i \omega t} \tilde{\Delta_{12}}\right\}
$$

und schließlich $\tilde{\Delta}_{22}=-\tilde{\Delta}_{11}$.

Auf dieselbe Weise wie früher erhält man daraus

$$
\begin{aligned}
& \tilde{\Delta}_{11}=-\frac{\frac{1}{2} \omega^{2} T_{1} T_{2} \delta_{1}{ }^{2} / \delta_{01}}{1+\left(\omega_{z}-\omega \delta_{0} / \delta_{01}\right)^{2} T_{2}{ }^{2}+\omega^{2} T_{1} T_{2} \delta_{1}{ }^{2} / \delta_{01}{ }^{2}} \\
& \tilde{\Delta}_{12}=-\frac{1}{2} i \omega \delta_{1} T_{2} \frac{1+i\left(\omega_{z}-\omega \delta_{0} / \delta_{01}\right) T_{2}}{1+\left(\omega_{z}-\omega \delta_{0} / \delta_{01}\right)^{2} T_{2}{ }^{2}+\omega^{2} T_{1} T_{2} \delta_{1}{ }^{2} / \delta_{01}{ }^{2}} e^{i \omega t} .
\end{aligned}
$$


Damit ist $\tilde{\Delta}(t)$ und $\tilde{\varrho}(t)$ bekannt. Mittels $\Delta=U^{+} \tilde{\Delta} U$ wird nun noch in die Ausgangsdarstellung zurück. transformiert, und man erhält

$$
\begin{aligned}
& \Delta_{11}=-\left[\frac{1}{2} \omega \delta_{1} \gamma H_{1} T_{2}{ }^{2}+\frac{1}{2} \omega^{2} \delta_{0} \frac{H_{1}{ }^{2}}{H_{0}{ }^{2}+H_{1}{ }^{2}} T_{2}\left(T_{1}-T_{2}\right)\right] \frac{1}{n}, \\
& \Delta_{12}=-\frac{1}{2} i \omega \delta_{1} T_{2}\left[1+i\left(\omega_{0}-\omega\right) T_{2}+i \omega \frac{H_{1}{ }^{2}}{H_{0}{ }^{2}+H_{1}{ }^{2}}\left(T_{1}-T_{2}\right)\right] \frac{1}{n} e^{i \omega t},
\end{aligned}
$$

wobei der Nenner $n$ gegeben ist durch

$$
n=1+\left(\omega_{0}-\omega\right)^{2} T_{2}^{2}+\gamma^{2} H_{1}^{2} T_{1} T_{2}+T_{2}\left(T_{1}-T_{2}\right)\left(\omega^{2} \frac{H_{1}{ }^{2}}{H_{0}{ }^{2}+H_{1}{ }^{2}}-\gamma^{2} H_{1}{ }^{2}\right) .
$$

Wir haben damit auch $\Delta(t)$ und $\varrho(t)$ und können als Anwendung wieder $\bar{M}_{x}$ berechnen:

$$
M_{x}=\chi_{0} H_{1} \cos \omega t+\chi_{0} \omega T_{2} H_{1} \frac{1}{n}\left\{\sin \omega t+\left[\left(\omega_{0}-\omega\right) T_{2}-\omega \frac{H_{1}{ }^{2}}{H_{0}{ }^{2}+H_{1}{ }^{2}}\left(T_{1}-T_{2}\right)\right] \cos \omega t\right\} .
$$

Mit (2.9 a) werden also jetzt die HF-Suszeptibilitäten

$$
\begin{aligned}
& \chi^{\prime}=\chi_{0}+\chi_{0} \omega T_{2} \frac{1}{n}\left[\left(\omega_{0}-\omega\right) T_{2}-\omega \frac{H_{1}{ }^{2}}{H_{0}{ }^{2}+H_{1}{ }^{2}}\left(T_{1}-T_{2}\right)\right], \\
& \chi^{\prime \prime}=\quad \chi_{0} \omega T_{2} \frac{1}{n} .
\end{aligned}
$$

Schließlich wird noch

$$
M_{z}=M_{z}{ }^{(0)}-\frac{1}{n} \chi_{0}\left[\omega \gamma H_{1}{ }^{2} T_{2}{ }^{2}+\omega^{2} H_{0} \frac{H_{1}{ }^{2}}{H_{0}{ }^{2}+H_{1}{ }^{2}} T_{2}\left(T_{1}-T_{2}\right)\right] .
$$

Für $T_{1}=T_{2}$ erhält man überall die entsprechenden Formeln von Kap. 3 zurück, ebenso für $H_{1} \rightarrow 0$. Damit gelten auch die Kronig-Kramers-Relationen für $H_{1} \rightarrow 0$, ferner ergeben sich für $H_{1} \rightarrow 0$ und $\omega_{0}=\gamma H_{0} \rightarrow 0$ die Debyeschen Formeln für $\chi^{\prime}$ und $\chi^{\prime \prime}$.

Für ein linear polarisiertes $H_{1}$-Feld führt eine entsprechende Rechnung wie oben, d. h. Übergang $\mathrm{zu}$ einem mit der Kreisfrequenz $\omega$ um die $y$-Achse schwingenden Koordinatensystem, nicht zum Ziel,

* Über die vorliegende Arbeit trug der Verfasser zum 21. Deutschen Physikertag in München (Sept. 1956) vor ${ }^{24}$, über Teile daraus sowie über einige experimentelle Untersuchungen bereits zur Physikertagung in Leipzig (April 1956) ${ }^{25}$.

24 G. Vojta, Phys. Verh. 7, 168 [1956].

25 G. Vojta, Exp. Techn. Phys. 4, 168 [1956].

A n m. b. d. K o r r. : R. K. Wangsness diskutiert in einer neuen Veröffentlichung (Phys. Rev. 104, 857 [1956]) die- da die Zeitabhängigkeit der Transformationsmatrix $U \mathrm{zu}$ verwickelt wird. Für diesen Fall kann man daher nur (4.9), (4.10) als eine Näherungslösung ansehen, diese wird aus der strengen Lösung wieder durch Vernachlässigen aller Glieder mit $\left(\omega_{0}+\omega\right)$ hervorgehen.

Der Verf. möchte Herrn Prof. Dr. Lösche, Leipzig, für die Stellung des Themas und Herrn Prof. Dr. Kockel, an dessen Institut die Arbeit * entstand, für Ratschläge herzlich danken.

jenigen Formen von verallgemeinerten BLochschen Gleichungen, die auf Grund des Prinzips der minimalen Entropieerzeugung bei stationären irreversiblen Prozessen möglich sind. Unsere Dichtematrix-Gleichungen sind auch von diesem Standpunkt aus korrekt. Für $T_{1} \neq T_{2}$ kann ein Relaxationsverhalten nach Gl. (85) von WANGSNess natürlich auch mit dem hier gewählten Verfahren (Abschnitt 3 , aber $\tau=T_{1}$ bzw. $T_{2}$ ) in einfacher Weise beschrieben werden. 